\title{
Unilateral Pulmonary Agenesis - As a cause of respiratory distress in newborn
}

\author{
Pradnya P. Wali ${ }^{1}$, Parakh $\mathbf{H}^{2}$, Reddy $\mathbf{K}^{3}$, Reddy $\mathbf{P}^{4}$ \\ ${ }^{1}$ Dr. Pradnya P. Wali, Junior Consultant, Neonatologist, ${ }^{2}$ Dr. Hemant Parakh, Consultant Neonatologist, ${ }^{3}$ Dr Krishna \\ Reddy, Consultant Pediatrician, ${ }^{4}$ Dr Prashant Reddy, Consultant Pediatrician, All authors are affiliated with Sunrise \\ Children Hospital, Hyderabad, India.
}

Address for Correspondence: Dr. Pradnya P. Wali, drpradnyawali@gmail.com.

\begin{abstract}
Pulmonary agenesis is a rare developmental defect which occurs as a result of failure of development of primitive lung bud. The onset \& mode of presentation is highly variable ranging from asymptomatic cases to cases diagnosed in adulthood due to recurrent respiratory tract infections. Around $50 \%$ of cases are associated with other congenital anomalies.Isolated U/L pulmonary agenesis, if not associated with other congenital abnormalities may have fewer symptoms \& long survival. Hereby we report a case of 2 days old female baby with right lung agenesis who presented with respiratory distress in neonatal period.
\end{abstract}

Key words: Lung, Unilateral, Agenesis, Newborn.

\section{Introduction}

Pulmonary agenesis is a rare congenital defect which was first described by De Pozze in 1673 [1,2]. In 1923, Muhammad reported first case of pulmonary agenesis from India [1,3]. It occurs as a result of failure of development of primitive lung bud due to abnormal blood flow in dorsal aortic arch during $4^{\text {th }}$ week of gestation. The onset \& mode of presentation is highly variable. Around $50 \%$ of cases are associated with other congenital anomalies, TEF \& VACTRAL in particular. Right sided agenesis have poorer prognosis due to its association with higher incidence of cardiac anomalies $[4,5,6]$. Isolated $\mathrm{U} / \mathrm{L}$ pulmonary agenesis, if not associated with other congenital abnormalities may have fewer symptoms \& long survival. We had this 2 days old female baby with right lung agenesis who presented with respiratory distress in neonatal period.

\section{Case Report}

A full term, $3.1 \mathrm{kgs}$, female baby delivered by Normal vaginal delivery was brought with h/o respiratory distress since birth. Baby cried immediately and had good apgars. Baby was admitted to NICU in view of distress \& was treated with IV Fluid, oxygen, antibiotics \& supportive treatment at local hospital. On day 2 of life baby was shifted to higher centre in view of persistent RD.

At admission: HR-160/min, RR- 70/min with mild subcostal \& intercostal retractions, Spo2 with oxygen was 98\%. Dull note was present on whole right chest with decreased breath sounds on right. Investigations sent showed Hb-11.9 gms/dl, TLC-35100, Platelets 1.61 lakhs, CRP- 98.5 (Strongly positive).

Blood c/s sent showed Enterobacter sepsis. Baby was treated with IVF, Oxygen, antibiotics according to sensitivity pattern. CSF analysis done was normal. Chest xray done showed opacification of Right lung with mediastinal shift to right.

Manuscript received: $8^{\text {th }}$ April 2017

Reviewed: $17^{\text {th }}$ April 2017

Author Corrected: $24^{\text {th }}$ April 2017

Accepted for Publication: 30 $30^{\text {th }}$ April 2017 

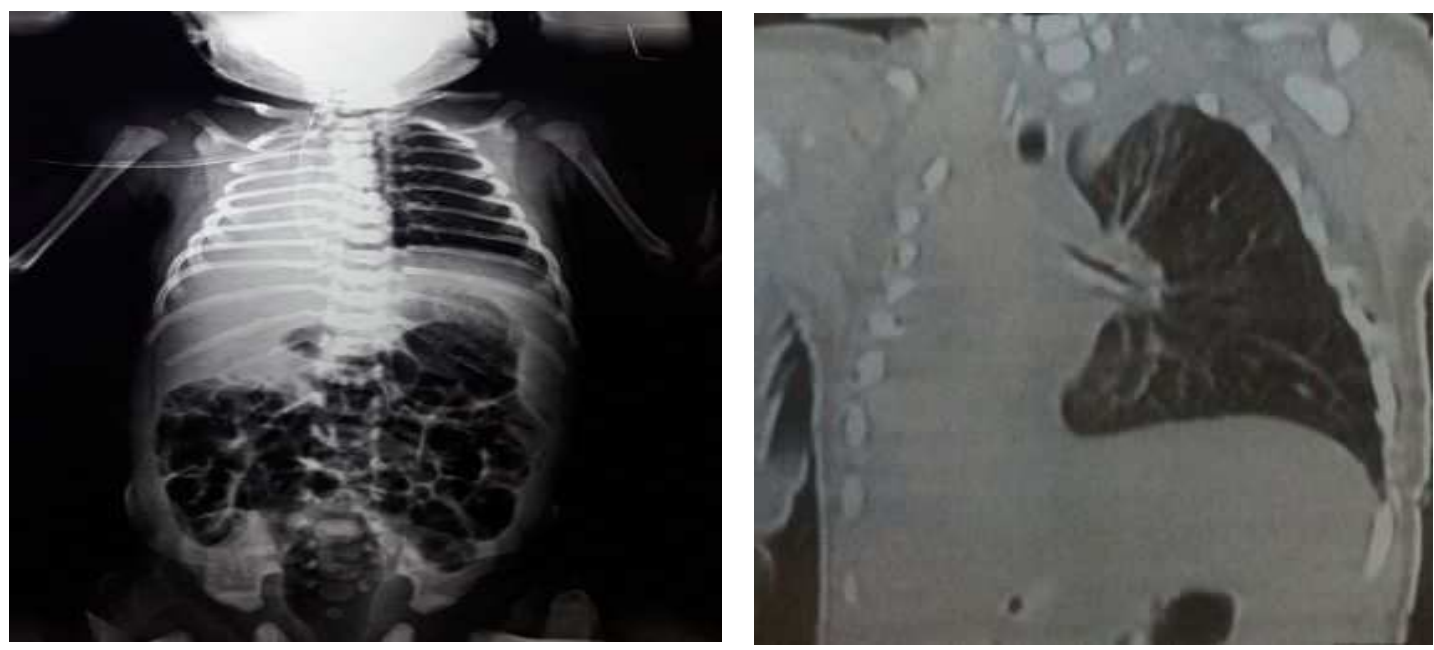

Fig-1: Chest x-ray showing complete opacification of Fig 2: CT with non visualization of right lung parenchyma right hemithorax with mediastinal shift to right $\quad \&$ ipsilateral pulmonary artery s/o Right lung agenesis.

NSG done was normal. 2D Echo showed 2 small muscular VSDs, L-R shunt, MPA-LPA, no PAH. Antibiotics were given for total 14 days. Baby improved clinically and was discharged in stable condition. Prevention of infections \& aggressive \& early treatment of bacterial infection with antibiotics was advised.

\section{Discussion}

Pulmonary agenesis is a rare developmental defect characterized by complete absence of lung tissues, bronchi \& pulmonary vessels with prevalence of 34 per million live births [7]. Pulmonary hypoplasia has decreased number or size of vessels \& alveoli but gross morphology of lung is preserved. It can be localized to single lobe, affects entire lung or rarely may be bilateral. B/L aplasia are not viable. U/L agenesis presents with varying degree of severity. The left lung is more affected than right $\&$ males are more affected than females [7].

Embryology: It occurs due to failure of development of respiratory system from foregut. Arrest at the stage of primitive lung bud produces $\mathrm{B} / \mathrm{L}$ pulmonary agenesis. The respiratory anlage at later stage produces U/L lung agenesis. Developmental arrest on one side in an older embryo results in lobar agenesis while failure of final alveolar differentiation during last trimester causes pulmonary hypoplasia [8].

Classification: Originally Classified by Schneider (1912) [9] \& then modified by Boyden (10) as:

1) Type 1- agenesis- complete absence of lung \& bronchus \& blood vessels on affected side.

2) Type 2- aplasia - rudimentary bronchus with complete absence of lung tissue.
3) Type 3- hypoplasia- presence of variable amounts of lung parenchyma, bronchial tree \& supporting vasculature.

Our case belonged to type 1 , as there was no lung tissue on affected side \& existing lung received the branch from the main pulmonary artery.

Etiopathogenesis: Genetic, Teratogenic, mechanical factors have been thought as etiological factors [8, 11]. Generally sporadic with autosomal recessive chromosomal aberration, consanguinity, intrauterine infections, VIT A deficiency, environmental factors are held responsible for agenesis $[8,12]$.

Clinical presentations: The presentation \& time of onset of symptoms is highly variable ranging from respiratory distress in neonatal period to recurrent pulmonary infections \& symptoms due to associated anomalies in later life.Sometimes U/L agenesis may be asymptomatic with accidental detection during routine examination. Our baby presented with respiratory distress since birth \& was being treated as a case of pneumonia as septic screen was positive \& chest $\mathrm{x}$-ray showing opacity on affected side.

Diagnosis: Can be diagnosed prenatally on ultrasound screening as hyperechoic hemithorax with prominent 
mediastinal shift.Chest $\mathrm{x}$ ray- dense homogenous opacity on affected side, raised hemi diaphragm, narrowed intercostals spaces \& heart shadow shifted to affected side [4,6] CT Chest confirms absent lung parenchyma \& pulmonary artery with ipsilateral mediastinal shift along with other congenital malformations if any [13]. Bronchoscopy \& pulmonary angiography is useful in demonstration of rudimentary bronchus \& absence of ipsilateral pulmonary vessel.cardiac catheterization may be required to quantify pulmonary artery pressure. But these tests are rarely performed due to their invasive nature $\&$ cost factor involved.

D/D: Pulmonary hypoplasia \& complete lung atelectasis in newborns. In adults collapse, thickened pleura, pneumonectomy.

Management: Asymptomatic cases need no intervention, but prevention of infection in solitary lung is very important as these infections can be life threatening. Hence these infections should be treated aggressively with antibiotics, bronchodilators \& physiotherapy [14].

Patients having stumps may require surgical removal if postural drainage \& antibiotics fails to resolve the infection. Corrective surgery of associated anomalies may be undertaken. Prophylaxis for RSV, Pneumococcus, influenza infections are recommended.

Prognosis: depends on two factors: 1) severity of associated congenital anomalies 2) involvement of normal lung in disease process. $(8,15)$ Mortality \& morbidity are related to associated complications \& death occurs due to progressive respiratory failure [4, 5]. $50 \%$ die at birth or within first 5 years of life [4,5]. Right lung agenesis has higher mortality than left lung agenesis. Asymptomatic cases \& those with minimal symptoms have good prognosis.

\section{Conclusion}

Pulmonary agenesis of right side without any other congenital anomaly as seen in our present case is extremely rare. Diagnosing a developmental lung anomaly in newborn is challenging for clinicians. Thus in symptomatic newborns presenting with RD with an opaque hemi thorax with ipsilateral mediastinal shift, rare condition like pulmonary agenesis should be considered \& thoroughly investigated.

\section{Funding: Nil, Conflict of interest: None Permission of IRB: Yes}

\section{References}

1. KH Kisku, MK Panigrahi. Agenesis of lung-a report of two cases. Lung India. Jan 2008; Vol 25, issue: 1, page 28-30.doi: 10.4103/0970-2113.44136.

2. De Pozze, Brescia MA, Amermon EE, Sharma K.K. Agenesis of the left Lung. Arch Pediatr.1960; 77: 485-490.

3. Muhamed K.S.N. Absence of left lung.Ind. Med. Gaz.1923:58:262-64.

4. K K Tan,C N Chin. Unilateral Pulmonary Agenesis an unusual cause of respiratory distress in the newborn. Singapore Med J 1996; Vol 37(6):668-669.

5. Kaya IS, Dilmen U. Agenesis of the lung. Eur Respir J. 1989 Jul;2(7):690-2.

6. Campanella C, Odell JA. Unilateral pulmonary agenesis. A report of 4 cases. S Afr Med J. 1987 Jun 20; 71 (12):785-7.

7. Roy PP, Datta S, Sarkar A, Das A, Das S. Unilateral pulmonary agenesis presenting in adulthood. Respir Med Case Rep. 2012;5:81-3. doi: 10.1016/j.rmedc. 2011.05.003. Epub 2011 Aug 10.

8. Shrestha P, Poudel P, Shah PL.Unilateral Pulmonary Aplasia. Case report. J.Nepal Paediatr.Soc. 2010;30 (2): 116-18.

9. Schneider P, Schawatbe E. Die Morphologic der Missbildungen Des Menschen under Thiere. Jena: Gustav Fischar. 1912; 3 Part.2:817-822.

10. Boyden EA. Developmental anomalies of the lungs. Am J Surg. 1955 Jan;89(1):79-89.

11. Finda JD, Michelson PH.Congenital disorders of the lung. Nelson Textbook of Pediatrics. $18^{\text {th }}$ Ed. Philadelphia: Saunders; 2008.p.1783.

12. Lee P, Westra S, Baba T, McCauley R.Right Pulmonary aplasia, aberrant left pulmonary artery \& bronchopulmonary sequestration with an esophageal bronchus. Pediatr Radiol. 2006 May, 36(5):449-52. Epub 2006 Mar 9. PMID: 16525768. 
13. Kravitz RM.Congenital malformations of the lung. Pediatr Clin North Am 1994, 41:453-72.

14. Singh U, Jhim D, Kumar S, Mittal V, Singh N, Gour $\mathrm{H}$, Ramaraj M. Unilateral agenesis of the lung: a rare entity. Am J Case Rep. 2015 Feb 8;16:69-72. doi: 10. 12659/AJCR.892385.

15. Chopra K, Sethi GR, Kumar A, Kapoor R, Saha MM, Mital M, Saluja S. Pulmonary agenesis. Indian Pediatr. 1988 Jul; 25(7):678-82.

\section{How to cite this article?}

Pradnya P. Wali, Parakh H, Reddy K, Reddy P. Unilateral Pulmonary Agenesis - As a cause of respiratory distress in newborn. Int J Med Res Rev 2017;5(04):442- 445. doi:10.17511/ijmrr. 2017.i04.11. 